\title{
Time To Administration Of First Antibiotics Is Associated With Hospital Length Of Stay In Children With Bacterial Infections
}

Sietske C. van Nassau ${ }^{1 *}$, Ron H. van Beek ${ }^{1}$, Gertjan J. Driessen², Herbert M. van Wering ${ }^{1}$, Navin P. Boeddha ${ }^{13,4}$

1. Department of Pediatrics, Amphia Hospital, Breda, The Netherlands

2. Department of Pediatrics, Juliana Children's Hospital/Haga Teaching Hospital, The Hague, The Netherlands

3. Intensive Care and Department of Pediatric Surgery, Erasmus MC-Sophia Children's Hospital, University Medical Center Rotterdam, Rotterdam, The Netherlands

4. Department of Pediatrics, Division of Pediatric Infectious Diseases \& Immunology, Erasmus MC-Sophia Children's Hospital, University Medical Center Rotterdam, Rotterdam, The Netherland

\section{Background}

Delay in antibiotic administration has been associated with increased mortality in adults with sepsis presenting to the emergency department (ED). Data in children are scarce and are skewed towards critically ill children.

\section{Aim}

We assessed the time to first antibiotics (TTFA) in children presenting to the ED with a bacterial infection, and studied the association with outcome.

\section{Methods}

Non-academic, single-center, retrospective study in children admitted with a bacterial infection, defined as receiving intravenous antibiotics within 24 hours after ED

registration with a subsequent in-hospital antibiotic therapy duration of at least 3 days $(n=485)$. Primary outcome measure was PICU transfer and/or mortality, and secondary outcome measure was prolonged hospital length of stay ( $\geq 7$ days).

\section{Results}

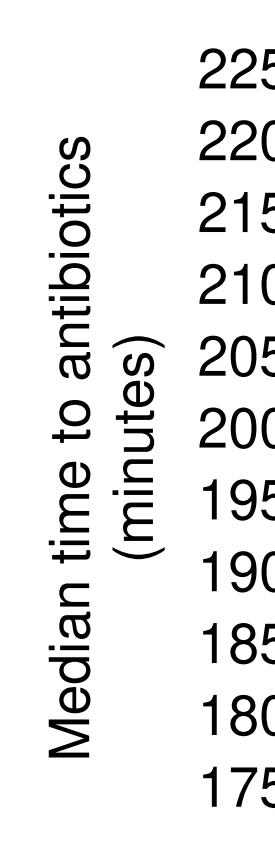

U transfer PICU transfer and/or mortality

Correlation TTFA and hospital LOS $r=0.104$

1500

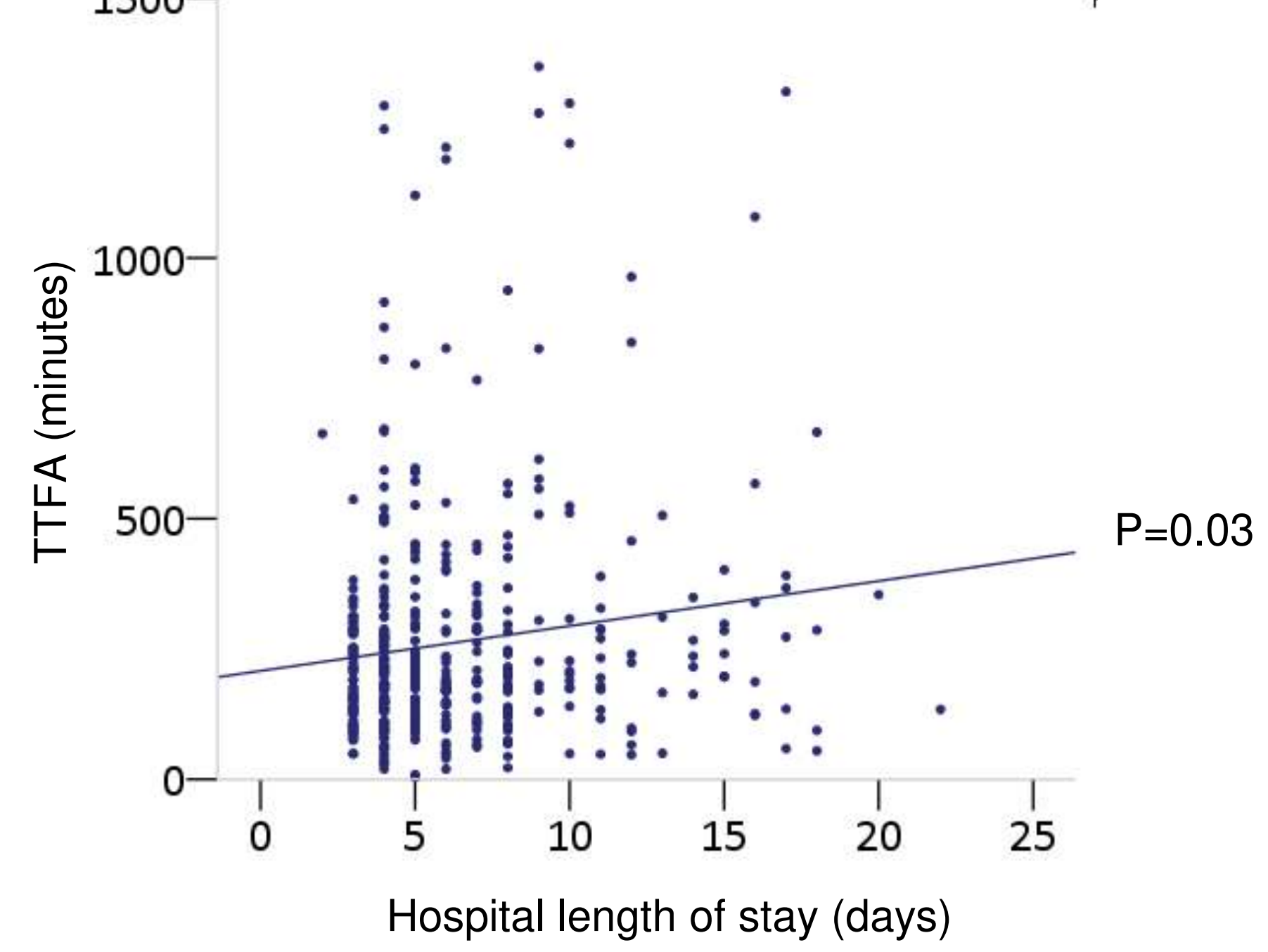

Amphit

\section{Discussion}

Conclusions

The time to first antibiotics was not associated with PICU transfer and/or death in this cohort of children presenting to the ED and subsequently admitted with a bacterial infection. We did find an association between TTFA and hospital length of stay.

\section{Future perspectives}

Larger, prospective multicenter studies adjusting for morbidity and illness severity are needed.

- Explore the utility of care bundles to reduce time to administration of first antibiotics. 